\title{
A Numerical Study of Blowup in the Harmonic Map Heat Flow Using the MMPDE Moving Mesh Method
}

\author{
Ronald D. Haynes ${ }^{1, *}$, Weizhang Huang ${ }^{2}$ and Paul A. Zegeling ${ }^{3}$ \\ ${ }^{1}$ Department of Mathematics and Statistics, Memorial University of \\ Newfoundland, St. John's, NL A1C 5S7, Canada. \\ ${ }^{2}$ Department of Mathematics, University of Kansas, Lawrence, KS 66045, USA. \\ ${ }^{3}$ Department of Mathematics, Utrecht University, Utrecht, The Netherlands.
}

Received October 30, 2011; Accepted (in revised version) 18 April 2012

Available online 12 April 2013

\begin{abstract}
The numerical solution of the harmonic heat map flow problems with blowup in finite or infinite time is considered using an adaptive moving mesh method. A properly chosen monitor function is derived so that the moving mesh method can be used to simulate blowup and produce accurate blowup profiles which agree with formal asymptotic analysis. Moreover, the moving mesh method has finite time blowup when the underlying continuous problem does. In situations where the continuous problem has infinite time blowup, the moving mesh method exhibits finite time blowup with a blowup time tending to infinity as the number of mesh points increases. The inadequacy of a uniform mesh solution is clearly demonstrated.
\end{abstract}

AMS subject classifications: 35K91, 65M50, 65M06

Key words: Heat flow, harmonic map, blowup, moving mesh method, finite difference.

\section{Introduction}

We are concerned with the numerical solution of the harmonic map heat flow into the unit sphere. In particular, we consider the initial-boundary value problem (IBVP)

$$
\begin{aligned}
& \theta_{t}=\theta_{r r}+\frac{1}{r} \theta_{r}-\frac{\sin (2 \theta)}{2 r^{2}}, \quad 0<r<1 \\
& \theta(0, t)=0, \quad \theta(1, t)=\theta_{1}, \\
& \theta(r, 0)=\theta^{0}(r), \quad 0<r<1,
\end{aligned}
$$

where $\theta^{0} \in C[0,1]$ is a given function satisfying $\theta^{0}(0)=0$ and $\theta^{0}(1)=\theta_{1}$. It is known $[12,17,18]$ that the solution of the IBVP (a) exists for all time if $\left|\theta^{0}(r)\right|<\pi$ for all

${ }^{*}$ Corresponding author. Email addresses: rhaynes@mun.ca (R. D. Haynes), huang@math.ku.edu (W. Huang), P.A.Zegeling@uu.nl (P. A. Zegeling) 
$r \in[0,1]$, (b) blows up in a finite time and at infinity if $\left|\theta_{1}\right|>\pi$ and $\left|\theta_{1}\right|=\pi$, respectively, and (c) may blow up in a finite time if $\left|\theta_{1}\right|<\pi$ but $\left|\theta^{0}(r)\right|$ rises above $\pi$ for some $r \in(0,1)$. Here, a solution is said to blow up in a finite time or at infinity (denoted by $T \in(0,+\infty]$ ) if

$$
\underset{t \uparrow T}{\limsup }\left\|\theta_{r}(\cdot, t)\right\|_{\infty}=+\infty .
$$

This is different from the widely studied blowup phenomenon associated with semilinear parabolic equations where the solution itself, instead of the spatial derivative of the solution, becomes unbounded in a finite time (e.g., see Friedman and Mcleod [25]). Moreover, via formal asymptotical analysis van den Berg et al. [35] show that for $\theta_{1}>\pi$, the blowup behavior of IBVP (1.1)-(1.3) is given by

$$
\lim _{t \uparrow T} \theta\left(\frac{\mu \kappa(T-t)}{|\ln (T-t)|^{2}}, t\right)=2 \arctan (\mu), \quad \text { for all fixed } \mu>0,
$$

where $\kappa>0$ is a constant and $\mu$ is called the kernel coordinate in the literature.

PDE (1.1) is a special case of the harmonic map heat flow

$$
u_{t}=\Delta u+|\nabla u|^{2} u,
$$

where $u(\cdot, t): D^{2} \rightarrow S^{2}, D^{2}$ is the unit disk in two dimensions and $S^{2}$ is the unit sphere in three dimensions. Indeed, it is easy to verify that (1.6) reduces to (1.1) for the radially symmetric solution

$$
u(r, \phi, t)=\left(\begin{array}{c}
\cos (\phi) \sin (\theta(r, t)) \\
\sin (\phi) \sin (\theta(r, t)) \\
\sin (\theta(r, t))
\end{array}\right),
$$

where $(r, \phi)$ are the polar coordinates for $D^{2}$ and $\theta(r, t)$ satisfies (1.1). PDE (1.6) is the gradient flow associated with the energy $\mathscr{E}=\frac{1}{2} \int_{D^{2}}|\nabla u|^{2} d V$ and its steady solution is a harmonic map from $D^{2}$ to $S^{2}$. It is known $[16,32]$ that IBVP (1.1)-(1.3) admits a classical solution for a sufficiently smooth initial solution with small energy and a global weak solution for a sufficiently smooth initial solution with finite energy. Such a weak solution is unique if the energy is non-increasing along the flow [24] and is smooth except for at most finitely many singular space-time points where non-constant harmonic maps "separate" and a downward jump in the energy and blowup in the spatial derivative of the solution occur. Finite time blowup in the harmonic map heat flow has been a topic of extensive research; e.g., see [12, 18, 20, 21, 26, 35].

PDEs (1.1) and (1.6) also arise in several other applications. For example, in the study of the evolution of the director field in nematic liquid crystals (LCs) [36], $u$ in (1.6) models the mean orientation of the long axis of the molecules comprising the LC, and (1.1) is obtained by considering LCs in a cylindrical tube. PDE (1.6) appears in the study of soft ferromagnets mechanically at rest $[13,22]$ where $u$ represents the magnetization of the deformable body. The harmonic map heat flow is also used in the study of color image enhancement or denoising; e.g., see $[33,38]$. 
The numerical solution of IBVP (1.1)-(1.3) has been considered by a number of researchers. In $[4,5,7]$, Bartels and his coworkers propose a fully discrete scheme for solving (1.6) and show the convergence to weak solutions of the continuous problem. They also give some computational evidence of blowup behavior, but no comparison to known analyses of the solution as the blowup time is approached. Their work is extended in [10] to harmonic map heat flow into non-constant target manifolds. Generalizations to $p$-harmonic heat flow and the Maxwell-Landau-Lifshitz-Gilbert equations are given in $[3,9]$ and $[1,8]$, respectively. The related problem of wave map into sphere, which has applications to general relativity, is considered in [6,7]. An adaptive finite element method is proposed by van der Schans [37] for solving a smoothed version of IBVP (1.1)-(1.3) (cf. Section 4.4).

We are interested in the numerical simulation of the blowup in the solution of IBVP (1.1)-(1.3) using a moving mesh method based on so-called moving mesh PDEs (MMPDEs) $[28,29]$. MMPDEs have been successfully used for the numerical simulation of blowup such as in semilinear parabolic equations [14, 15, 19, 27], Cahn-Hilliard equations [23], and integro-differential equations [31]. In those situations, the solution becomes unbounded and the numerical computation typically stops at a finite time despite the use of a uniform or an adaptive mesh $[2,11]$. On the other hand, the blowup with (1.1) is different, occurring in the spatial derivative instead of the solution itself. This may make its numerical simulation more difficult. Indeed, proper mesh adaptation is necessary so that the minimal mesh spacing can become infinitesimal and the corresponding spatial derivative of the solution can become infinitely large. As we shall see in Section 4, a uniform mesh will not lead to an infinitely large spatial derivative and the computation typically takes a small time step for a while and then continues to reach a steady state solution.

A main objective of this paper is to investigate how well MMPDEs work for the numerical simulation of the blowup in IBVP (1.1)-(1.3). We restrict our attention to MMPDE5 (cf. (2.3)), a commonly used MMPDE. Using the blowup behavior (1.5) and a properly chosen monitor function, we show that MMPDE5 defines a coordinate transformation (or equivalently an adaptive mesh) under which the blowup profile of the solution can be accurately represented. Moreover, we establish asymptotic forms as $t \rightarrow T$ for the coordinate transformation and the solution and its spatial derivative. These asymptotics are verified by the numerical results obtained with the MMPDE5-based moving mesh method which is truly adaptive and makes no use of the blowup behavior (1.5). The agreement between the theoretical prediction and the numerics in turn provides confidence in the moving mesh method. The method is then used for the study of the blowup of (1.1) for various initial conditions and that of the degree- $m$ equivariant map generalization of (1.1). Numerical results show that the method leads to finite time blowup when the underlying continuous problem does. In situations where the continuous problem has infinite time blowup, the method exhibits finite time blowup with the blowup time tending to infinity as the number of mesh points increases.

The paper is arranged as follows. MMPDE5 and the related moving mesh method for solving (1.1)-(1.3) are described in Section 2. An asymptotic analysis is given in Section 3 for MMPDE5 with a properly chosen monitor function and blowup behavior (1.5). In Sec- 
tion 4 the method is applied to the study of the blowup in IBVP (1.1)-(1.3) for various initial conditions. The extension of the numerical study to the degree- $m$ equivariant harmonic map generalization of (1.1) is given in Section 5. We conclude in Section 6 with a summary and some comments.

\section{Moving mesh PDE method}

A moving finite difference method based on a moving mesh PDE (MMPDE) $[29,30]$ is used for the numerical study of IBVP (1.1)-(1.3). With the method, a mesh of $J+1$ points,

$$
r_{0}(t)=0<r_{1}(t)<\cdots<r_{J}(t)=1,
$$

is generated through a coordinate transformation $r=r(\xi, t)$ from the computational domain $\Omega_{c}=(0,1)$ to the physical domain $\Omega=(0,1)$; i.e.,

$$
r_{j}(t)=r\left(\xi_{j}, t\right), \quad j=0, \cdots, J,
$$

where $\xi_{j}=j / J, j=0, \cdots, J$ is a uniform mesh on $\Omega_{c}$. Such a coordinate transformation $r=r(\xi, t)$ is determined by an MMPDE and suitable boundary conditions. Here we use the so-called MMPDE5 [29]

$$
\tau \dot{r}=\frac{\partial}{\partial \xi}\left(M \frac{\partial r}{\partial \xi}\right), \quad \xi \in(0,1)
$$

subject to the boundary conditions

$$
r(0, t)=0, \quad r(1, t)=1,
$$

where "." denotes the time derivative in the new coordinates $\xi$ and $t, M=M(\theta, r, t)$ is a suitably chosen monitor function, depending on the solution $\theta$ and used to control mesh concentration, and $\tau>0$ is a constant parameter used to tune the response of the mesh movement to the changes in $M$. The choice of $M$ and $\tau$ has been discussed extensively in the literature, e.g., see $[30,34]$. In our computation, we take $\tau=10^{-2}$ and

$$
M=\left(1+\left(\theta_{r}\right)^{2}\right)^{\frac{\gamma}{2}}
$$

for some constant $\gamma>1$; see the analysis in the next section which motivates this choice of $\gamma$.

Once $M$ has been chosen, the unknown variable $\theta(\xi, t)$ and the coordinate transformation $r(\xi, t)$ can be obtained by numerically solving PDE (1.1) and moving mesh PDE (2.3) simultaneously. To this end, we employ the method of lines approach and use central finite differences for spatial discretization. Specifically, a central finite difference discretization of (2.3) reads as

$$
\tau \dot{r}_{j}=\frac{1}{\Delta \xi^{2}}\left[M_{j+1 / 2}\left(r_{j+1}-r_{j}\right)-M_{j-1 / 2}\left(r_{j}-r_{j-1}\right)\right], \quad j=1, \cdots, J-1,
$$


where $\Delta \xi=1 / J$ and $M_{j+1 / 2}$ is a finite difference approximation of $M$ in (2.5) at $r=$ $r_{j+1 / 2}=\left(r_{j}+r_{j+1}\right) / 2$. Since the so-computed monitor function is typically non-smooth, it is common practice in the context of moving mesh methods to smooth $M$ for a smoother mesh and easier integration of the moving mesh PDE. In our computation, the monitor function is smoothed using ten sweeps of a low-pass filter (cf. [30]).

For the discretization of PDE (1.1), we first transform from the physical coordinate $r$ to the computational coordinate $\xi$. Using the chain rule, we have

$$
\theta_{t}=\dot{\theta}-\dot{r} \theta_{r}, \quad \theta_{r}=\frac{\theta_{\xi}}{r_{\xi}} .
$$

Then we rewrite (1.1) as

$$
\dot{\theta}-\frac{\dot{r}}{r_{\xi}} \theta_{\xi}=\frac{1}{r_{\xi}}\left(\frac{\theta_{\xi}}{r_{\xi}}\right)_{\xi}+\frac{\theta_{\xi}}{r r_{\xi}}-\frac{\sin (2 \theta)}{2 r^{2}} .
$$

Letting $\theta_{j} \approx \theta\left(r_{j}(t), t\right)$ and discretizing the above PDE using central finite differences, we get

$$
\begin{aligned}
\dot{\theta}_{j}-\dot{r}_{j} \frac{\theta_{j+1}-\theta_{j-1}}{r_{j+1}-r_{j-1}}= & \frac{2}{r_{j+1}-r_{j-1}}\left(\frac{\theta_{j+1}-\theta_{j}}{r_{j+1}-r_{j}}-\frac{\theta_{j}-\theta_{j-1}}{r_{j}-r_{j-1}}\right) \\
& +\frac{\theta_{j+1}-\theta_{j-1}}{r_{j}\left(r_{j+1}-r_{j-1}\right)}-\frac{\sin \left(2 \theta_{j}\right)}{2 r_{j}^{2}}, \quad j=1, \cdots, J-1 .
\end{aligned}
$$

Eqs. (2.6) and (2.8) and suitable approximations of boundary conditions (1.2) and (2.4) form a system of differential-algebraic equations (DAE) which is solved using the Matlab DAE solver ode15i. In our computation, the relative and absolute tolerances for the adaptive time integration (used in ode15i) are taken to be reltol $=10^{-8}$ and abstol $=$ $10^{-6}$.

\section{An asymptotic analysis for MMPDE5}

In this section we give a formal analysis of the moving mesh method described in the previous section. We want to identify the values of $\gamma$ for which MMPDE5 (2.6) works satisfactorily with the monitor function defined as

$$
M=\left(\theta_{r}\right)^{\gamma} .
$$

This monitor function is essentially the same as that in (2.5) especially for $r \approx 0$ since $\theta_{r}(0, t)$ becomes very large as $t \rightarrow T$. We follow the approach of $[15,27]$ to solve the MMPDE analytically using the exact form (1.5) for the solution. Note that this approach is not practical in general since the physical solution is what is sought by the computation. Nevertheless, the analysis determines the "optimal" mesh for our particular problem, which can be used to verify our numerical results. Moreover, the approach is for theoretical 
analysis only. In actual computation, the monitor function is calculated using the computed solution and the resulting mesh is thus truly adaptive. The numerical results obtained this way in Section 4 will be compared to the theoretical findings obtained in this section.

We start the analysis by rewriting (1.5) as

$$
\theta(\mu R(t), t)=2 \arctan (\mu)+o(1), \quad \text { as } t \rightarrow T,
$$

where

$$
R(t)=\frac{\kappa(T-t)}{|\ln (T-t)|^{2}}
$$

Noticing that the blowup profile is characterized in (3.2) in the kernel coordinate $\mu=$ $r / R(t)$, we would expect that it can be accurately represented in a computational coordinate $\xi$ which is a smooth function of $\mu$. Thus, we seek a coordinate transformation $r=r(\xi, t)$ in the form

$$
r(\xi, t)=R(t) z(\xi, t)
$$

with the property

$$
z(\xi, t)=z_{0}(\xi)+O(\tau)+o(1), \quad \text { as } t \rightarrow T,
$$

where $o(1)$ denotes terms tending to zero as $t \rightarrow T$ and $z_{0}(\xi)$ is to be determined. The existence of such a coordinate transformation is used to judge whether or not MMPDE5 (2.3) works satisfactorily for the current problem.

Differentiating (3.4) with respect to $t$ and $\xi$, we have

$$
\begin{aligned}
& \dot{r}=R(t) \dot{z}+\dot{R}(t) z, \\
& r_{\xi}=R(t) z_{\xi}, \\
& r_{\xi \xi}=R(t) z_{\xi \xi},
\end{aligned}
$$

where

$$
\dot{R}(t)=-\frac{\kappa}{|\ln (T-t)|^{2}}+\frac{2 \kappa}{(\ln (T-t))^{3}} .
$$

Moreover, differentiating (3.2) with respect to $\mu$ gives

$$
\theta_{r}(\mu R(t), t) R(t)=\frac{2}{1+\mu^{2}}+o(1), \quad \text { as } t \rightarrow T
$$

or

$$
\theta_{r}(\mu R(t), t)=\frac{2}{1+\mu^{2}} \frac{1}{R(t)}+o\left(\frac{1}{R(t)}\right), \quad \text { as } t \rightarrow T
$$

Using (3.1) and (3.10), we get

$$
M=\left(\frac{2}{1+z^{2}}\right)^{\gamma} \frac{1}{R(t)^{\gamma}}+o\left(\frac{1}{R(t)^{\gamma}}\right),
$$

from which we find

$$
M_{\xi}=\frac{-2^{\gamma+1} \gamma}{\left(1+z^{2}\right)^{\gamma+1}} \frac{z z_{\xi}}{R(t)^{\gamma}}+o\left(\frac{1}{R(t)^{\gamma}}\right)
$$


Substituting the above results into MMPDE (2.3) leads to

$$
\tau[R(t) \dot{z}+\dot{R}(t) z]=\frac{2^{\gamma} z_{\xi \xi}}{\left(1+z^{2}\right)^{\gamma}} \frac{1}{R(t)^{\gamma-1}}+\frac{2^{\gamma}(-2 \gamma) z z_{\xi}^{2}}{\left(1+z^{2}\right)^{\gamma+1}} \frac{1}{R(t)^{\gamma-1}}+o\left(\frac{1}{R(t)^{\gamma-1}}\right),
$$

or, after some simplification,

$$
\tau\left[R(t)^{\gamma} \dot{z}+R(t)^{\gamma-1} \dot{R}(t) z\right]=\left[z_{\xi \xi}-\frac{2 \gamma z z_{\xi}}{1+z^{2}}\right] \frac{2^{\gamma}}{\left(1+z^{2}\right)^{\gamma}}+o(1) .
$$

From (3.3) and (3.9), it is easy to see that, for $\gamma>1$,

$$
R(t)^{\gamma-1} \dot{R}(t) \rightarrow 0, \quad \text { as } t \rightarrow T .
$$

When $\gamma>1$, the right-hand-side terms of (3.13) dominate over the left-hand-side terms as $t \rightarrow T$. Since these terms correspond to the right-hand-side or equidistribution term of (2.3), the MMPDE is said to have dominance of equidistribution when they dominate. MMPDEs with the property of equidistribution dominance are shown in [27] to admit a coordinate transformation in the form of (3.4) with property (3.5) for semilinear parabolic PDEs. For the current situation, it is easy to see that $z_{0}(\xi)$ satisfies

$$
z_{0}^{\prime \prime}-\frac{2 \gamma z_{0}\left(z_{0}^{\prime}\right)^{2}}{1+z_{0}^{2}}=0 \text {. }
$$

This equation is supplemented with the boundary conditions

$$
z_{0}(0)=0, \quad \lim _{\xi \rightarrow 1^{-}} z_{0}(\xi)=\infty
$$

which correspond to the boundary conditions $r(0, t)=0$ and $r(1, t)=1$ for $r=r(\xi, t)$ (cf. (2.4)). Notice that the second condition corresponds to the limit of large $z_{0}$ (or $z$ ) for $r=r(\xi, t)$ in the form (3.4); the interested reader is referred to a more detailed discussion on the boundary conditions in $[15,27]$.

It is interesting to point out that (3.15) is similar to the corresponding equation (cf. Eq. (32) of [27]) for blowup of semilinear parabolic equations. This is because the monitor function $M$, defined in (3.1), has an asymptotic form (3.11) which is similar to that (cf. Eq. (28) of [27]) of semilinear parabolic equations where $M$ is defined as a power of the physical solution.

Boundary value problem (3.15) and (3.16) can be solved by letting $v=d z_{0} / d \xi$ and separating variables. This yields

$$
\xi=\frac{\int_{0}^{z_{0}(\xi)}\left(1+s^{2}\right)^{-\gamma} d s}{\int_{0}^{\infty}\left(1+s^{2}\right)^{-\gamma} d s}
$$

The integrals in the above equation can be expressed in terms of the Gauss hypergeometric function for general $\gamma$. For $\gamma=3 / 2$, they have analytical expressions

$$
\int_{0}^{z_{0}}\left(1+s^{2}\right)^{-\frac{3}{2}} d s=\frac{z_{0}}{\sqrt{1+z_{0}^{2}}}, \quad \int_{0}^{\infty}\left(1+s^{2}\right)^{-\frac{3}{2}} d s=1,
$$


and $z_{0}$ can be found as

$$
z_{0}(\xi)=\frac{\xi}{\sqrt{1-\xi^{2}}} .
$$

For this value of $\gamma$, the coordinate transformation can be written as

$$
r(\xi, t)=R(t)\left[\frac{\xi}{\sqrt{1-\xi^{2}}}+O(\tau)+o(1)\right], \quad \text { as } t \rightarrow T
$$

and the blowup profile of the solution can be expressed in the computational coordinate as

$$
\theta(\xi, t)=2 \arctan \frac{\xi}{\sqrt{1-\xi^{2}}}+O(\tau)+o(1), \quad \text { as } t \rightarrow T .
$$

Moreover, from (3.10) we can see

$$
\left\|\theta_{r}(\cdot, t)\right\|_{\infty}=\left|\theta_{r}(0, t)\right|=\frac{2}{R(t)}, \quad \text { as } t \rightarrow T .
$$

Thus, using $\left\|\theta_{r}(\cdot, t)\right\|_{\infty}$ we can rewrite $\theta_{r}(\xi, t)$ and $r(\xi, t)$ as

$$
\begin{aligned}
& \frac{\left|\theta_{r}(\xi, t)\right|}{\left\|\theta_{r}(\cdot, t)\right\|_{\infty}}=1-\xi^{2}+O(\tau)+o(1), \quad \text { as } t \rightarrow T, \\
& r(\xi, t)=\frac{2}{\left\|\theta_{r}(\cdot, t)\right\|_{\infty}}\left[\frac{\xi}{\sqrt{1-\xi^{2}}}+O(\tau)+o(1)\right], \quad \text { as } t \rightarrow T .
\end{aligned}
$$

The asymptotic behavior (3.20), (3.22), and (3.23) will be verified in the next section through numerical examples.

\section{Numerical experiments}

In this section, we present numerical results for the blowup in the solution of IBVP (1.1)-(1.3) for various initial conditions. We are interested in the formation, time, and profile of the blowup and if they can be correctly simulated numerically. Both uniform and adaptive moving meshes are used. For mesh adaptation, we employ the moving mesh PDE method described in Section 2 along with MMPDE5 (2.3), $\tau=10^{-2}$, and monitor function (2.5) with $\gamma=3 / 2$. It is emphasized that the derivative needed in (2.5) is approximated by a divided finite difference of the computed solution and the computational procedure is truly adaptive.

\subsection{Finite time blowup case: $\theta_{1}>\pi$}

We begin by considering an initial condition

$$
\theta^{0}(r)=4.25 \pi r^{2}
$$




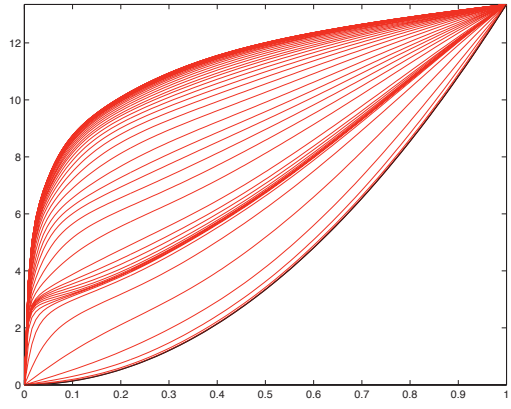

(a) $\theta$ vs. $r$, uniform mesh $J=128$

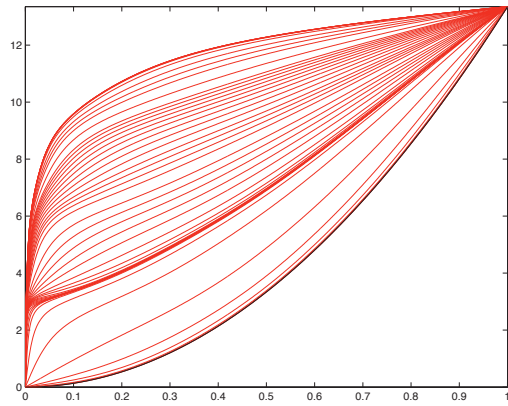

(c) $\theta$ vs. $r$, uniform mesh $J=512$

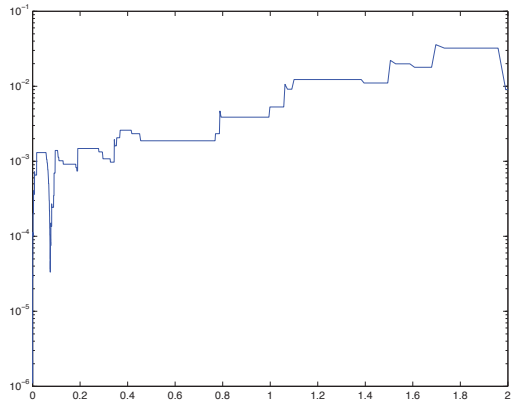

(b) $\Delta t$ vs. $t$, uniform mesh $J=128$

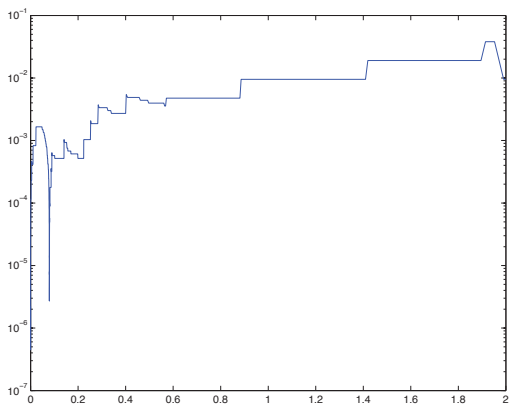

(d) $\Delta t$ vs. $t$, uniform mesh $J=512$

Figure 1: IBVP (1.1)-(1.3) with the initial solution (4.1) on uniform meshes. The black curves in (a) and (c) are the initial solution.

with $\theta_{1}=4.25 \pi>\pi$. Theoretically, we expect finite time blowup in $\theta_{r}$. Snapshots of the solution at a sequence of time instants obtained with a uniform mesh of points $J=128$ and $J=512$ are shown in Fig. 1 . They show that the computation takes a small time step $\left(\Delta t \approx 3 \times 10^{-5}\right.$ ) near $t=0.08$ while the computational solution is developing a large spatial derivative at $r=0$. However, after a while, the apparent development of blowup stops and the integration continues with a much larger step size and finally the solution seems to reach a steady state. The same phenomenon occurs when a larger number of mesh points is used (a solution with $J=512$ is shown) although a much smaller time step size ( $\Delta t \approx 3 \times 10^{-6}$, corresponding to a larger spatial derivative) is used for the finer mesh. These results indicate that the central finite difference scheme on a uniform mesh fails to reproduce the theoretically predicted finite time blowup.

Results obtained on a moving mesh of $J=128$ points are shown in Fig. 2 and 4. Unlike the uniform mesh situation, the blowup develops near $t=0.08238$, where the spatial derivative at the origin increases dramatically and reaches $10^{9}$. Near this time, the minimal spacing of the mesh and the time step size for the ODE integrator are getting smaller and finally reach around $10^{-11}$ and $10^{-16}$, respectively. Note that the integration cannot be carried passing the blowup time; it stops because the allowed minimum time step (about the size of machine epsilon) is reached (cf. Fig. 4(d)). The solution $\theta$ is plotted as a 


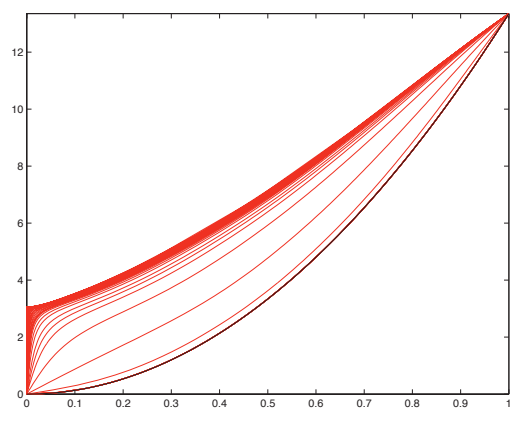

(a) $\theta$ vs. $r$

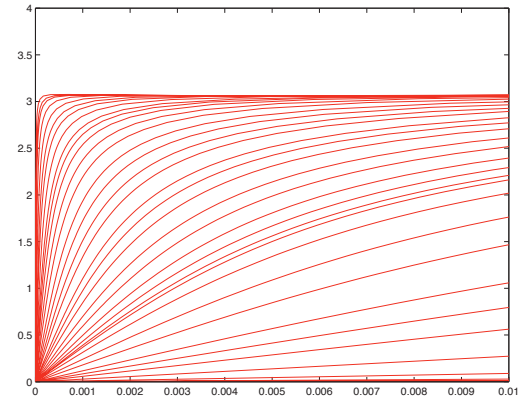

(b) close up near $r=0$

Figure 2: IBVP (1.1)-(1.3) with the initial solution (4.1) on a moving mesh of $J=128$. The solution $\theta$ is plotted as a function of $r$ for a number of time instants. The black curve is the initial solution.

function of $r$ in Fig. 2 for various time instants. It can be seen that the solution has a very sharp gradient at $r=0$ especially when $t$ is close to the blowup time. A moving mesh of 41 points is shown in Fig. 3.

Figs. 4 (a) and (b) show $\theta(\xi, t)$ and $\theta_{r}(\xi, t) /\left\|\theta_{r}(\cdot, t)\right\|_{\infty}$ as functions of $\xi$ for various time instants. The green lines are the corresponding asymptotic values for the quantities given in (3.20) and (3.22). The figures show that the computational approximations of these quantities are approaching their asymptotics as $t \rightarrow T$. They can be made closer with finer meshes, see Fig. 5. These agreements consolidate our theoretical analysis in the previous section and also provide confidence in our numerical results. To further demonstrate the ability of the moving mesh method to represent the blowup profile accurately in the computational coordinate $\xi$, we choose $\xi=\xi_{j}$ in (3.23) and take the logarithm of the both sides of the resulting equation. We have

$$
\ln r_{j} \approx-\ln \left(\left\|\theta_{r}(\cdot, t)\right\|_{\infty}\right)+\ln \left(\frac{2 \xi_{j}}{\sqrt{1-\xi_{j}^{2}}}\right), \quad \text { as } t \rightarrow T \text {. }
$$

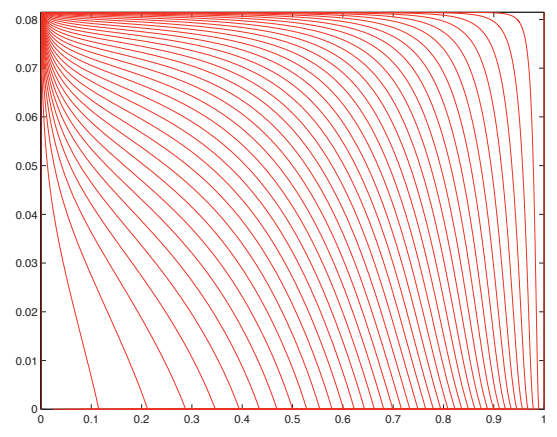

Figure 3: An adaptive moving mesh of 41 points is shown for IBVP (1.1)-(1.3) with the initial solution. The $x$ and $y$ axes are $r$ and $t$, respectively. 


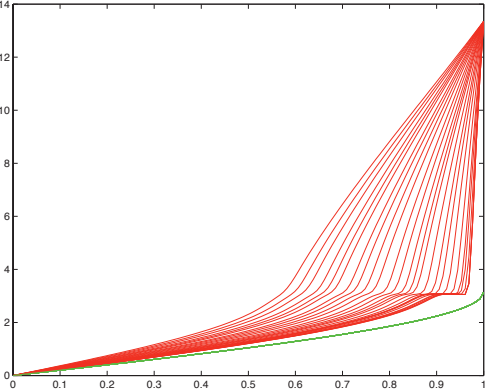

(a) $\theta$ vs. $\xi$

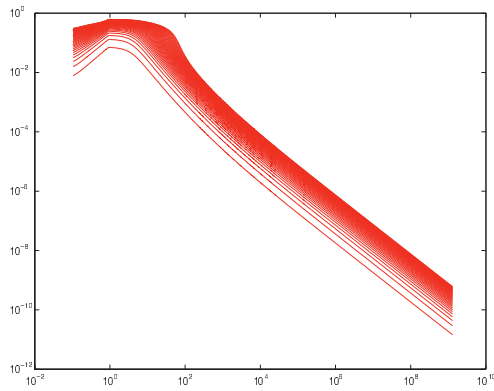

(c) $r_{j}$ vs. $\left\|\theta_{r}(\cdot, t)\right\|_{\infty}$

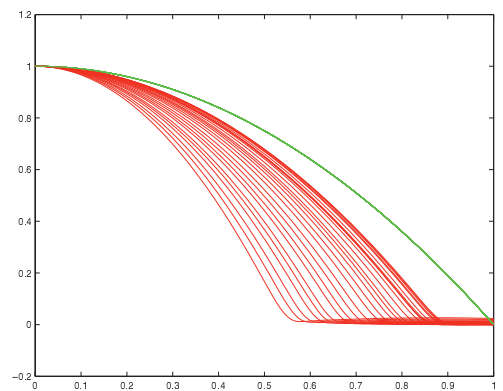

(b) $\theta_{r} /\left\|\theta_{r}(\cdot, t)\right\|_{\infty}$ vs. $\xi$

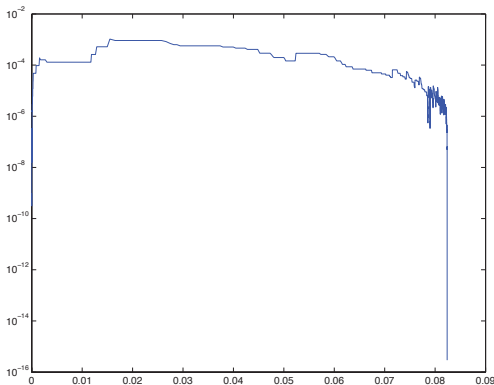

(d) $\Delta t$ vs. $t$

Figure 4: IBVP (1.1)-(1.3) with the initial solution (4.1) on a moving mesh of $J=128$. The green curves in (a) and (b) are the corresponding asymptotic values (3.20) and (3.22), respectively.

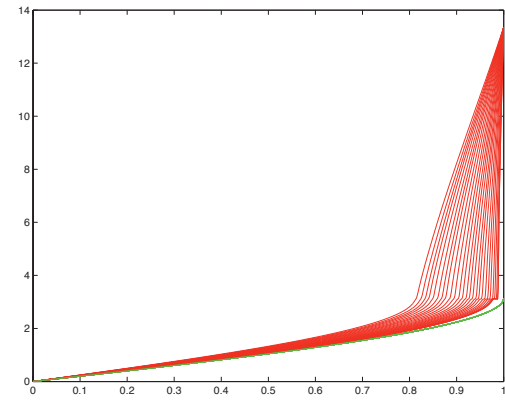

(a) $\theta$ vs. $\xi$

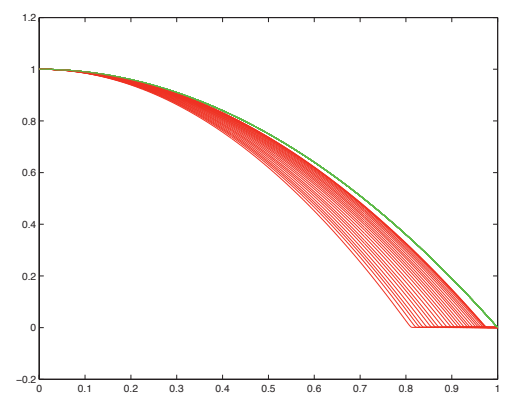

(b) $\theta_{r} /\left\|\theta_{r}(\cdot, t)\right\|_{\infty}$ vs. $\xi$

Figure 5: IBVP (1.1)-(1.3) with the initial solution (4.1) on a moving mesh of $J=1024$. The green curves in (a) and (b) are the corresponding asymptotic values (3.20) and (3.22), respectively.

This implies that for a given $j, \ln r_{j}$ is a linear function of $\ln \left(\left\|\theta_{r}(\cdot, t)\right\|_{\infty}\right)$ as $t \rightarrow T$ and for $r_{j}$ close to the origin. This is verified in Fig. 4(c) where $\ln r_{j}$ is shown as a function of $\ln \left(\left\|\theta_{r}(\cdot, t)\right\|_{\infty}\right)$ for $j=2, \cdots, 40$.

The blowup time is plotted as a function of $J$ in Fig. 6 . One can see that the blowup 


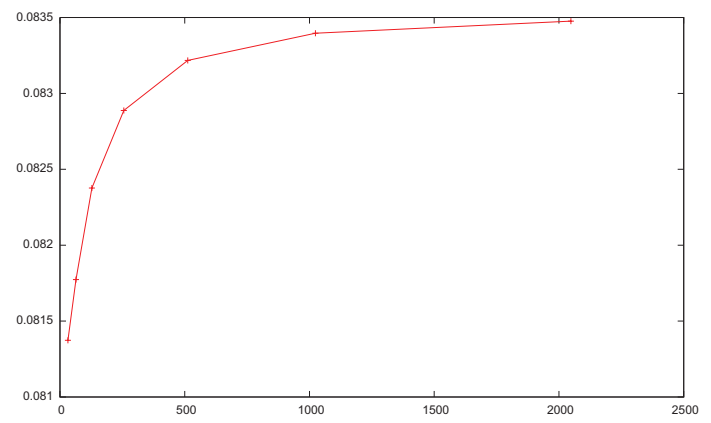

Figure 6: IBVP (1.1)-(1.3) with the initial solution (4.1) on moving meshes. Blowup time $T$ is plotted as a function of $J$.

time is converging to a finite time as $J \rightarrow \infty$. This suggests that the continuous problem has finite time blowup, consistent with the formal analysis of [35].

Finally, we consider an initial condition with two peaks,

$$
\theta^{0}(r)=\pi \sin (2 \pi r)+2 \pi r .
$$

Results obtained with the moving mesh method are shown in Fig. 7. As in the case with initial condition (4.1), the computational solution develops blowup at a finite time and is approaching to the asymptotic described in (3.22) for the blowup region (near the origin). For the remote region (near the outside boundary), the solution tends to match the outside boundary condition while maintaining the two-peak shape of the initial condition.

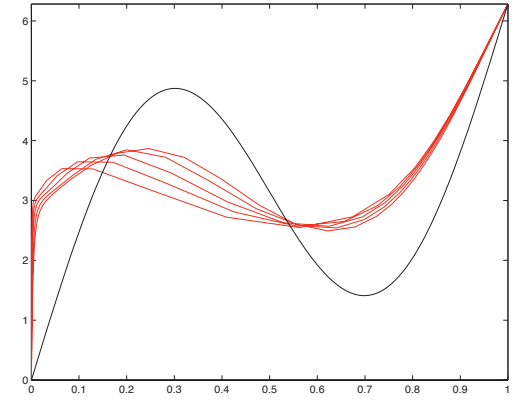

(a) $\theta$ vs. $r$

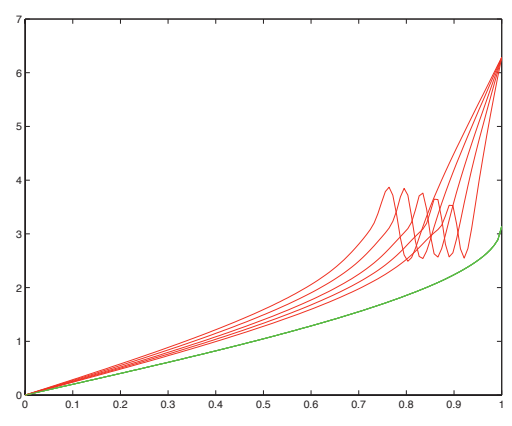

(b) $\theta$ vs. $\xi$

Figure 7: IBVP (1.1)-(1.3) with the initial solution (4.3) on a moving mesh of $J=128$. The black curve in (a) is the initial solution. The green curve in (b) is the asymptotic value (3.20).

\subsection{Borderline case: $\theta_{1}=\pi$}

For this borderline case, it is known theoretically that blowup occurs at infinity. To study this situation, we consider the initial solution

$$
\theta^{0}(r)=\pi r^{2}
$$




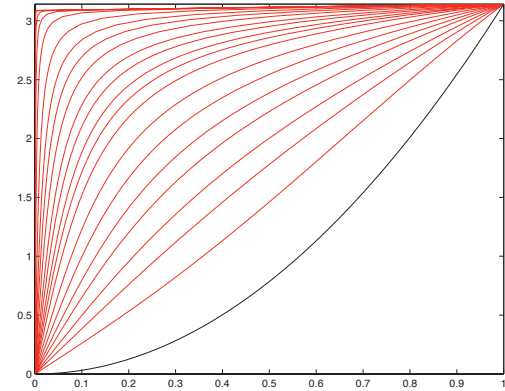

(a) $\theta$ vs. $r$

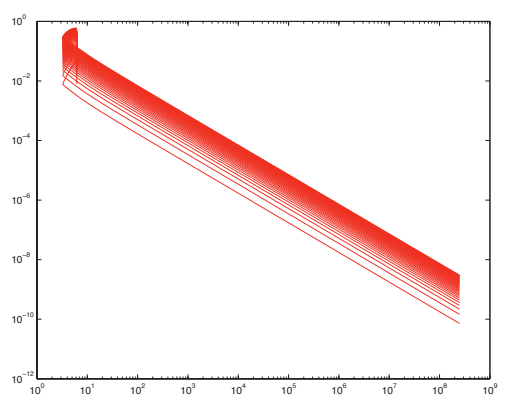

(c) $r_{j}$ vs. $\left\|\theta_{r}(\cdot, t)\right\|_{\infty}$

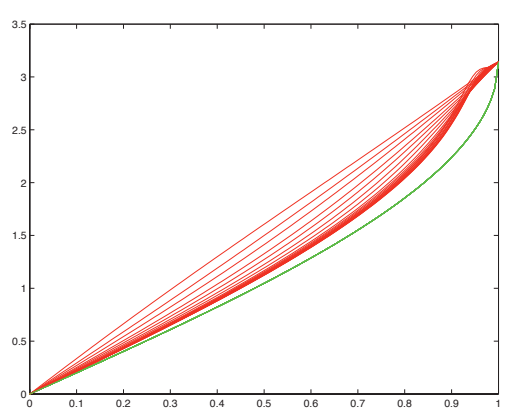

(b) $\theta$ vs. $\xi$

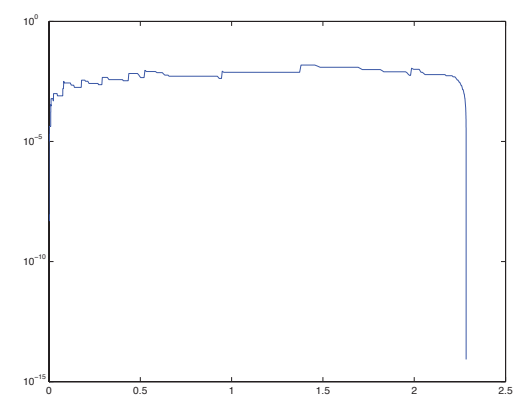

(d) $\Delta t$ vs. $t$

Figure 8: IBVP (1.1)-(1.3) with the initial solution (4.4) on a moving mesh of $J=128$. The black curve in (a) is the initial solution and the green curve in (b) is the asymptotic value (3.20).

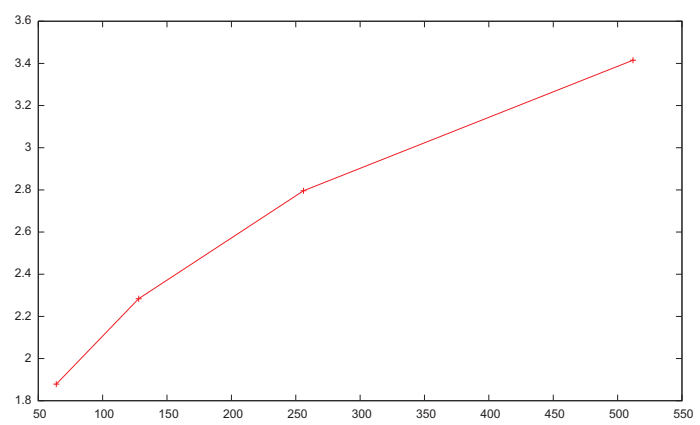

Figure 9: IBVP (1.1)-(1.3) with the initial solution (4.4) on moving meshes. Blowup time $T$ is plotted as a function of $J$.

Results obtained with the moving mesh method are shown in Fig. 8. Surprisingly, the computational solution for a fixed $J=128$ does not blow up at infinity. Instead, it blows up at a finite time $(T \approx 2.284)$ and exhibits almost the same behavior as the case discussed in Section 4.1; see and compare Figs. 8 and 4. The same phenomenon holds for larger $J$ (results not shown here). However, the blowup time does not seem to converge to a finite value as $J$ increases; instead, it seems to converge to infinity, see Fig. 9. This can be viewed as a reflection of the infinity time blowup of the underlying continuous problem. 
4.3. Initial conditions with $\theta_{1}<\pi$ and $\theta^{0}(r)$ rising above $\pi$ for some $r \in(0,1)$

For this type of initial condition, the solution may or may not blow up (at a finite time). To see this, we consider the initial condition

$$
\theta^{0}(r)=\alpha \pi\left((1-r) \sin (2 \pi r)+\frac{1}{2} r\right),
$$

where $\alpha$ is a parameter. Results are shown in Fig. 10 for $\alpha=1.5$ and $\alpha=1.6$. Finite time blowup occurs for $\alpha=1.6$ but not with $\alpha=1.5$. Interestingly, from Fig. 10(c) and (d) one can see that the solution for $\alpha=1.5$ develops a sharp gradient and appears to approach the asymptotics, however, this development eventually ceases and the solution converges to a steady state which is a monotone function connecting boundary conditions $\theta(0)=0$ and $\theta(1)=\pi$.

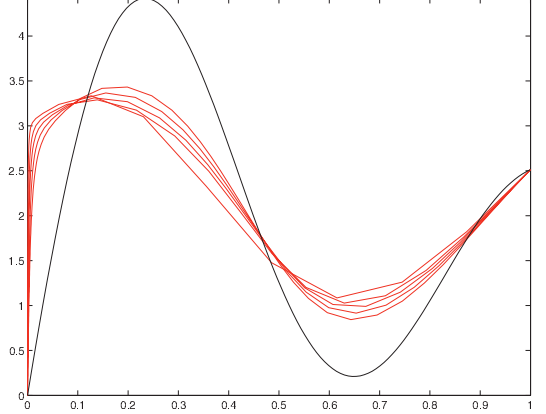

(a) $\theta$ vs. $r, \alpha=1.6$

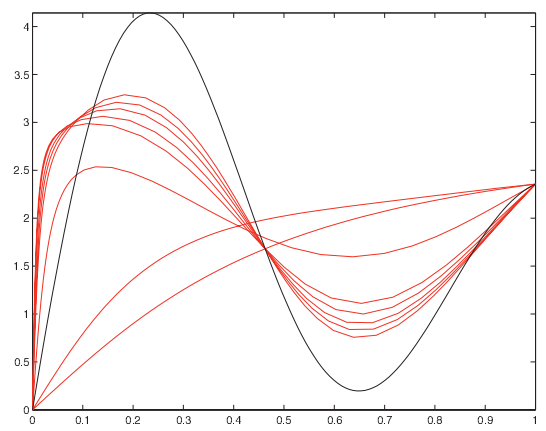

(c) $\theta$ vs. $r, \alpha=1.5$

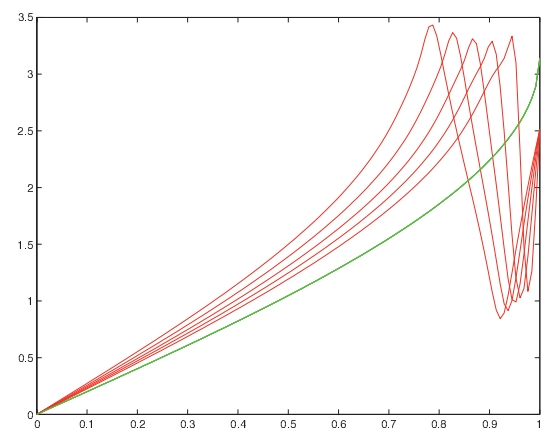

(b) $\theta$ vs. $\xi, \alpha=1.6$

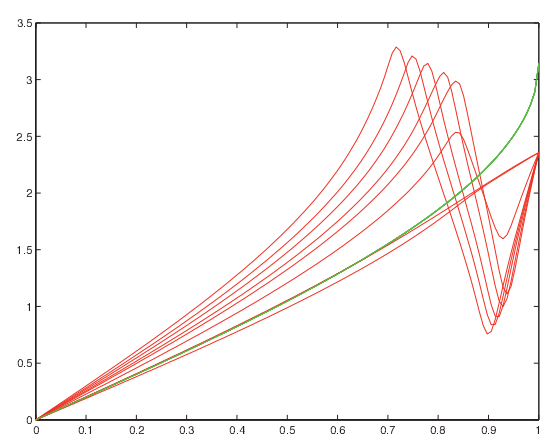

(d) $\theta$ vs. $\xi, \alpha=1.5$

Figure 10: IBVP (1.1)-(1.3) with the initial solution (4.5) on a moving mesh of $J=128$. The black curves in (a) and (c) are the initial solutions while the green curves in (b) and (d) are the asymptotic value (3.20).

\subsection{A smoothed problem}

Recall that PDE (1.1) is singular at $r=0$. A regularization method is used by van der Schans [37] for the finite element solution of IBVP (1.1)-(1.3). The regularization is 
achieved by modifying the physical domain from $(0,1)$ to $(\epsilon, 1)$ for some small $\epsilon>0$. The smoothed IBVP reads as

$$
\begin{cases}\theta_{t}=\theta_{r r}+\frac{1}{r} \theta_{r}-\frac{\sin (2 \theta)}{2 r^{2}}, & \epsilon<r<1, \\ \theta(\epsilon, t)=0, \quad \theta(1, t)=\theta_{1}, & \\ \theta(r, 0)=\theta^{0}(r), & \epsilon<r<1 .\end{cases}
$$

Following [37], we consider an initial condition

$$
\theta^{0}(r)=\pi(2 \sin (\pi(r-\epsilon))+0.2(r-\epsilon))
$$

for which $\theta_{1}<\pi$ but $\theta^{0}(r)$ arises above $\pi$ for some $r \in(\epsilon, 1)$. Note that the original IBVP (without smoothing) with this initial condition has a finite time blowup around $t=0.0162$. Numerical solutions obtained with the moving mesh method for IBVP (4.6) with $\epsilon=10^{-5}$ are shown in Fig. 11. One can see that the computational solution develops a large gradient and appears to initially approach the asymptotic solution (3.20) but instead ultimately reaches a steady state. This phenomenon is similar to what observed in [37]. Our results seem to suggest that the regularization can change the dynamics of the underlying problem dramatically.

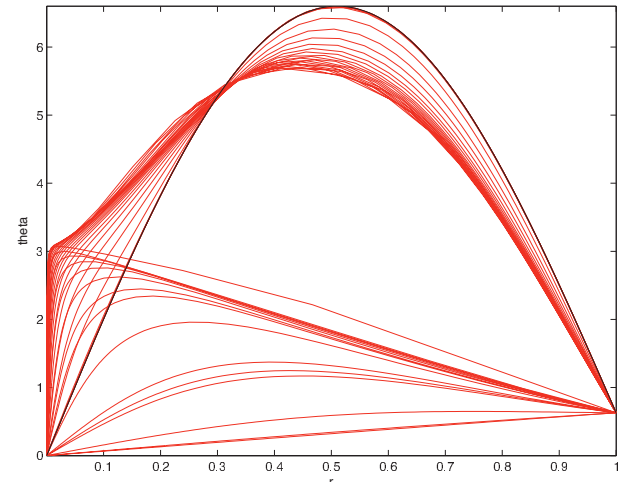

(a) $\theta$ vs. $r$

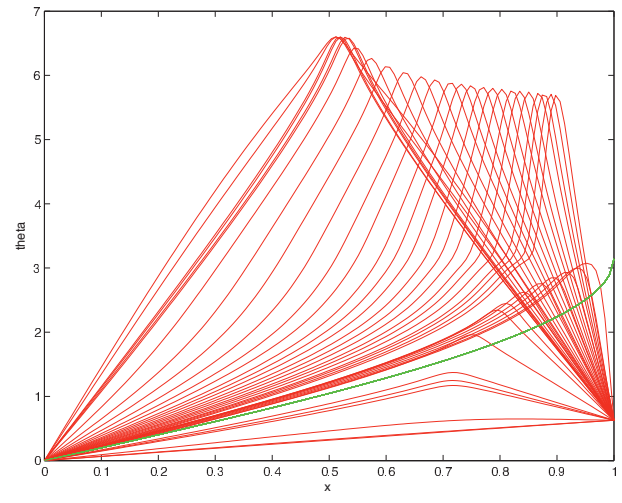

(b) $\theta$ vs. $\xi$

Figure 11: Smoothed IBVP on a moving mesh of $J=128$. The black curve in (a) is the initial solution and the green curve in (b) is the asymptotic solution (3.20).

\section{Degree- $m$ equivariant harmonic maps}

PDE (1.1) belongs to a family of so-called degree-m equivariant maps (e.g., see [26]) governed by

$$
\theta_{t}=\theta_{r r}+\frac{1}{r} \theta_{r}-m^{2} \frac{\sin (2 \theta)}{2 r^{2}}, \quad 0<r<1,
$$


where $m$ is a positive number. The above PDE is obtained by seeking radially symmetric solutions of the form

$$
u(r, \phi, t)=\left(\begin{array}{c}
\cos (m \phi) \sin (\theta(r, t)) \\
\sin (m \phi) \sin (\theta(r, t)) \\
\sin (\theta(r, t))
\end{array}\right),
$$

in PDE (1.6). The dynamics of (5.1) are very different from those of (1.1) especially for $m \geq 2$. In particular, it is known [35] that PDE (5.1) can only have infinity time blowup when $m \geq 2$.

The blowup behavior for IBVP (5.1), (1.2), and (1.3) is given by

$$
\underset{t \uparrow T}{\limsup } \theta(R(t) \mu, t)=2 \arctan \left(\mu^{m}\right), \quad \text { for all fixed } \mu>0,
$$

where $T$ can be finite or infinity and $R(t)$ is defined such that

$$
R(t)^{m} \theta(r, t) \sim 2 r^{m}, \quad \text { as } r \rightarrow 0 \text { and as } t \rightarrow T .
$$

(The interested reader is referred to [35] for the expression of $R(t)$ for all different cases of $m$.) The above relation suggests that we can choose the monitor function in the form

$$
M(r, t)=\left(\frac{\partial \theta^{\frac{1}{m}}}{\partial r}\right)^{\gamma}
$$

for some $\gamma>0$. With this choice of the monitor function, $\|M(\cdot, t)\|_{\infty} \sim R(t)^{-\gamma}$ and analysis can be done as in Section 3 for MMPDE5 (2.3). Indeed, using the properties

$$
R(t) \rightarrow 0, \quad R(t)^{\gamma-1} \dot{R}(t) \rightarrow 0, \quad \text { as } t \rightarrow T,
$$

we can show that MMPDE5 has the dominance of equidistribution when $\gamma>1$. In the case, a coordinate transformation in the form (3.4) and (3.5) exists, with $z_{0}(\xi)$ determined by

$$
\left\{\begin{array}{l}
z_{0}^{\prime \prime}+\gamma\left(z_{0}^{\prime}\right)^{2}\left[\frac{(m-1) z_{0}^{m-1}}{\arctan \left(z_{0}^{m}\right)}+\frac{m-1-(m+1) z_{0}^{2 m}}{\left(1+z_{0}^{2 m}\right) z_{0}}\right]=0 \\
z_{0}(0)=0, \quad \lim _{\xi \rightarrow 1^{-}} z_{0}(\xi)=\infty
\end{array}\right.
$$

Fig. 12 shows the results obtained with the moving mesh method with monitor function (5.5) $(\gamma=3 / 2)$ for PDE (5.1) with $m=4$ and the initial solution

$$
\theta^{0}(r)=1.5 \pi r^{2} \text {. }
$$

As for the borderline case discussed in Section 4.2, the computational solution for a fixed $J$ also shows a finite time blowup behavior but the blowup time, as shown in Fig. 13, is tending to infinity as $J \rightarrow \infty$. The latter suggests that the continuous problem has blowup at infinity, which is consistent with the theoretical analysis.

Finally, we point out that solving (5.1) with a uniform mesh does not lead to blowup at a finite time or infinity. Moreover, the maximal derivative of the computational solution does not occur at the origin but instead at a small distance from the origin; cf. Fig. 14(a). The distance decreases as $J$ increases, see Fig. 14(b). 


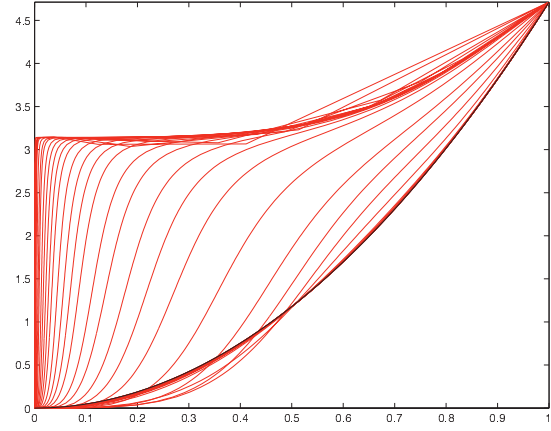

(a) $\theta$ vs. $r$

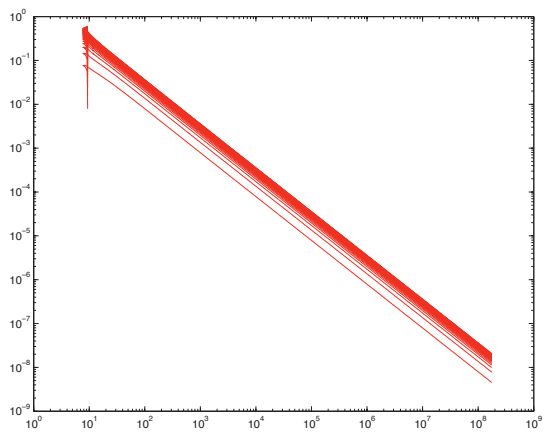

(c) $r_{j}$ vs. $\left\|\theta_{r}(\cdot, t)\right\|_{\infty}$

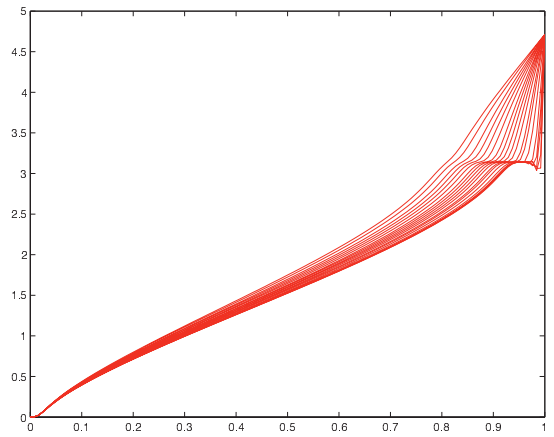

(b) $\theta$ vs. $\xi$

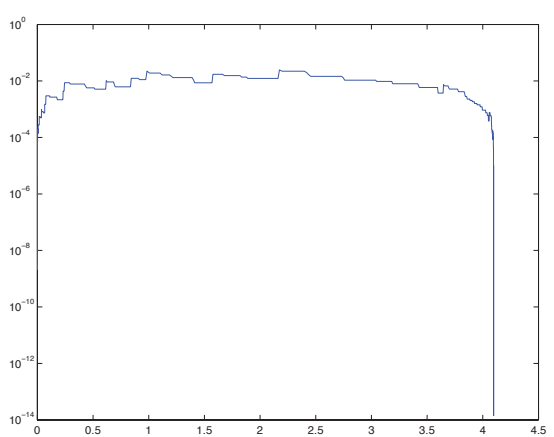

(d) $\Delta t$ vs. $t$

Figure 12: IBVP (5.1) $(m=4),(1.2)$, and (1.3) with the initial solution (5.8) on a moving mesh of $J=128$. The black curve in (a) is the initial solution.

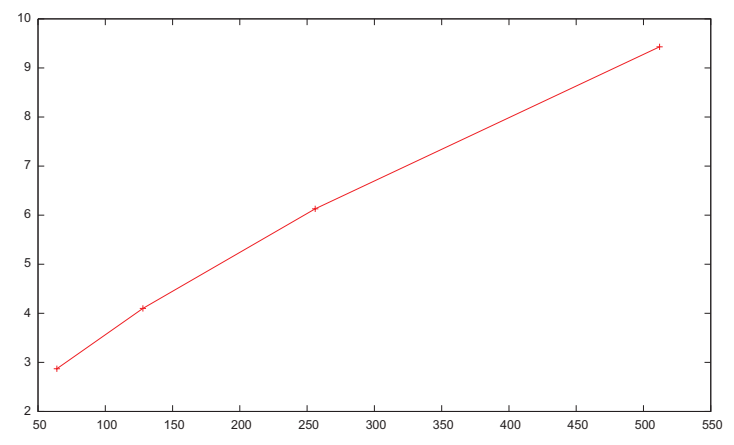

Figure 13: IBVP (5.1) $(m=4),(1.2)$, and (1.3) with the initial solution (5.8) on moving meshes. Blowup time $T$ is plotted as a function of $J$.

\section{Conclusions and comments}

In the previous sections we have studied the moving mesh simulation of the blowup in the harmonic map heat flow (1.1) or more generally (5.1) with $m>0$. Using the blowup 


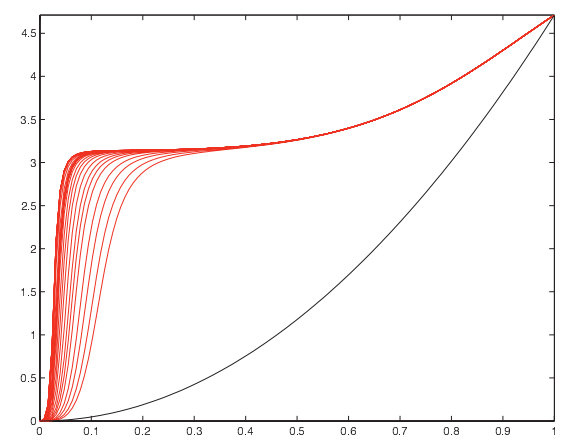

(a) $\theta$ vs. $r(J=128)$

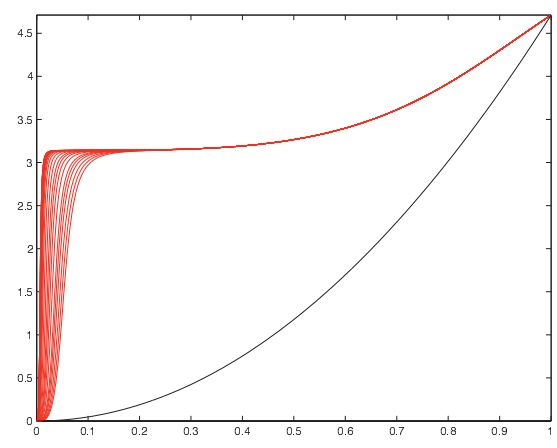

(b) $\theta$ vs. $r(J=512)$

Figure 14: IBVP (5.1) $(m=4),(1.2)$, and (1.3) with the initial solution (5.8) on a uniform mesh. The black curves are the initial solution.

profile (1.5) or (5.3), we have shown that MMPDE5 (2.3), together with monitor function (3.1) or (5.5), admits a coordinate transformation in the form (3.4) and (3.5) with $z_{0}(\xi)$ satisfying (3.15) or (5.7). For the special case with $m=1$ and $\gamma=3 / 2$, the coordinate transformation is found to have the form (3.19) and the solution $\theta$ and its derivative $\theta_{r}$ are given in (3.20) and (3.23) in the computational coordinate $\xi$. These asymptotics have been used to verify the moving mesh method described in Section 2 and in turn verified by numerical results obtained with the method.

Numerical results presented in Sections 4 and 5 have demonstrated that with a properly chosen monitor function, the moving mesh method can be used to simulate the blowup in the harmonic map heat flow and produce accurate blowup profiles. More specifically, the computational solution has a finite time blowup and the blowup time converges to a finite value for the situation where the continuous problem has finite time blowup. On the other hand, for the situation when the continuous problem has blowup at infinity, the moving mesh scheme for a fixed number $(J)$ of mesh points exhibits a finite time blowup but the blowup time tends to infinity as $J$ tends to infinity. For both situations, the numerical approximations can be made closer to their asymptotics by using a larger $J$.

Numerical results also show that uniform meshes cannot produce "real" blowup. Indeed, for cases where the continuous problem has finite time blowup or blowup at infinity, the solutions on a uniform mesh first develop sharp gradient at or near the origin and then this development stops and finally tend to steady state solutions. The phenomenon holds when a finer mesh is used. Moreover, for the degree- $m$ equivariant map case with $m \geq 2$, the maximal derivative of the computed solution does not occur at the origin but instead at a small distance from the origin. This distance decreases as a finer mesh is used.

It should be pointed out that the analysis and the moving mesh computation presented in the previous sections do not rely on the explicit knowledge of $R(t)$, the scaling factor defined in (5.4). On one hand, this indicates that the MMPDE moving mesh method is robust for use in numerical simulation of different types of blowup. On the other hand, it also implies that our numerical results may not be very useful in predicting or verifying 
$R(t)$. Numerical prediction or verification of $R(t)$ is certainly a topic deserving further investigations. It is also worth mentioning that applications of the current work to actual problems in liquid crystals, ferromagnetism and image processing and the extension to $2 \mathrm{D}$ harmonic map heat flow are currently under investigation.

Acknowledgments This work was supported in part by NSF (U.S.A.) under grants DMS0712935 and DMS-1115118 and by NSERC (Canada) under discovery grant 311796.

\section{References}

[1] L. Baňas, S. Bartels, and A. Prohl. A convergent implicit finite element discretization of the Maxwell-Landau-Lifshitz-Gilbert equation. SIAM J. Numer. Anal., 46:1399-1422, 2008.

[2] C. Bandle and H. Brunner. Blowup in diffusion equations: a survey. J. Comput. Appl. Math., 97:3-22, 1998.

[3] J. W. Barrett, S. Bartels, X. Feng, and A. Prohl. A convergent and constraint-preserving finite element method for the $p$-harmonic flow into spheres. SIAM J. Numer. Anal., 45:905-927, 2007.

[4] S. Bartels. Combination of global and local approximation schemes for harmonic maps into spheres. J. Comput. Math., 27:170-183, 2009.

[5] S. Bartels. Numerical analysis of a finite element scheme for the approximation of harmonic maps into surfaces. Math. Comp., 79:1263-1301, 2010.

[6] S. Bartels, X. Feng, and A. Prohl. Finite element approximations of wave maps into spheres. SIAM J. Numer. Anal., 46:61-87, 2007/08.

[7] S. Bartels, C. Lubich, and A. Prohl. Convergent discretization of heat and wave map flows to spheres using approximate discrete Lagrange multipliers. Math. Comp., 78:1269-1292, 2009.

[8] S. Bartels and A. Prohl. Convergence of an implicit finite element method for the LandauLifshitz-Gilbert equation. SIAM J. Numer. Anal., 44:1405-1419 (electronic), 2006.

[9] S. Bartels and A. Prohl. Convergence of an implicit, constraint preserving finite element discretization of $p$-harmonic heat flow into spheres. Numer. Math., 109:489-507, 2008.

[10] L. Baňas, A. Prohl, and R. Schätzle. Finite element approximations of harmonic map heat flows and wave maps into spheres of nonconstant radii. Numer. Math., 115:395-432, 2010.

[11] M. Berger and R. V. Kohn. A rescaling algorithm for the numerical calculation of blowing-up solutions. Comm. Pure Appl. Math, 41:841-863, 1988.

[12] M. Bertsch, R. Dal Passo, and R. van der Hout. Nonuniqueness for the heat flow of harmonic maps on the disk. Arch. Ration. Mech. Anal., 161(2):93-112, 2002.

[13] M. Bertsch, P. Podio-Guidugli, and V. Valente. On the dynamics of deformable ferromagnetic solids: I. Global weak solutions for soft ferromagnets at rest. Ann. Mat. Pura Appl., 179:331360, 2001.

[14] C. J. Budd, V. A. Galaktionov, and J. F. Williams. Self-similar blow-up in higher-order semilinear parabolic equations. SIAM J. Appl. Math., 64:1775-1809 (electronic), 2004.

[15] C. J. Budd, W. Huang, and R. D. Russell. Moving mesh methods for problems with blow-up. SIAM J. Sci. Comput., 17:305-327, 1996.

[16] K.-C. Chang. Heat flow and boundary value problem for harmonic maps. Ann. Inst. H. Poincaré Anal. Non Linéaire, 6:363-395, 1989.

[17] K.-C. Chang and W.-Y. Ding. A result on the global existence for heat flows of harmonic maps from $D^{2}$ into $S^{2}$. In Nematics (Orsay, 1990), volume 332 of NATO Adv. Sci. Inst. Ser. C Math. Phys. Sci., pages 37-47. Kluwer Acad. Publ., Dordrecht, 1991. 
[18] K.-C. Chang, W.-Y. Ding, and R. Ye. Finite-time blow-up of the heat flow of harmonic maps from surfaces. J. Diff. Geom., 36:507-515, 1992.

[19] S. H. Chen. A sufficient condition for blowup solutions of nonlinear heat equations. J. Math. Anal. Appl., 293:227-236, 2004.

[20] Y. Chen and W.-Y. Ding. Blow-up and global existence for heat flows of hamronic maps. Invent. Math., 99:567-578, 1990.

[21] J.-M. Coron and J.-M. Ghidaglia. Explosion en temps fini pour le flot des applications harmoniques. C. R. Acad. Sci. Paris Sér. I Math., 308:339-344, 1989.

[22] A. DeSimone and P. Podio-Guidugli. On the continuum model of deformable ferromagnetic solids. Arch. Ration. Mech. Anal., 136:201-233, 1996.

[23] J. D. Evans, V. A. Galaktionov, and J. F. Williams. Blow-up and global asymptotics of the limit unstable Cahn-Hilliard equation. SIAM J. Math. Anal., 38:64-102 (electronic), 2006.

[24] A. Freire. Uniqueness of the harmonic map flow from surfaces to general targets. Comment. Math. Helv., 70:310-338, 1995.

[25] A. Friedman and B. Mcleod. Blow up of positive solutions of semilinear heat equations. Indiana Univ. Math. J., 34:425-447, 1985.

[26] M. Guan, S. Gustafson, and T.-P. Tsai. Global existence and blow-up for harmonic map heat flow. J. Diff. Eq., 246:1-20, 2009.

[27] W. Huang, J. Ma, and R. D. Russell. A study of MMPDE moving mesh methods for the numerical simulation of blowup in reaction diffusion equations. J. Comput. Phys., 227:65326552, 2008.

[28] W. Huang, Y. Ren, and R. D. Russell. Moving mesh methods based on moving mesh partial differential equations. J. Comput. Phys., 113:279-290, 1994.

[29] W. Huang, Y. Ren, and R. D. Russell. Moving mesh partial differential equations (MMPDEs) based upon the equidistribution principle. SIAM J. Numer. Anal., 31:709-730, 1994.

[30] W. Huang and R. D. Russell. Adaptive Moving Mesh Methods. Springer, New York, 2011. Applied Mathematical Sciences Series, Vol. 174.

[31] J. Ma, Y. Jiang, and K. Xiang. On a moving mesh method for solving partial integrodifferential equations. J. Comput. Math., 27:713-728, 2009.

[32] M. Struwe. On the evolution of harmonic mappings of Riemannian surfaces. Comment. Math. Helv., 60:558-581, 1985.

[33] B. Tang, G. Sapiro, and V. Casselles. Color image enhancement via chromaticity diffusion. IEEE Trans. Image Proc., 10:701-707, 2001.

[34] A. van Dam and P. A. Zegeling. Balanced monitoring of flow phenomena in moving mesh methods. Comm. Comput. Phys., 7:138-170, 2010.

[35] J. B. van den Berg, J. Hulshof, and J. R. King. Formal asymptotics of bubbling in the harmonic map heat flow. SIAM J. Appl. Math., 63:1682-1717, 2003.

[36] R. van der Hout. Flow alignment in nematic liquid crystals in flows with cylindrical symmetry. Diff. Int. Eq., 14:189-211, 2001.

[37] M. van der Schans. Harmonic map heat flow. Technical report, Mathematical Institute Leiden, 2006. Master Thesis.

[38] L. A. Vese and S. J. Osher. Numerical methods for $p$-harmonic flows and applications to image processing. SIAM J. Numer. Anal., 40:2085-2104, 2002. 